\title{
A hardware tool for explained power electronics control of induction motors
}

\author{
Rui Esteves Araújo*, Henrique Teixeira**, José Barbosa** and Vicente Leite** \\ * FACULDADE DE ENGENHARIA DA UNIVERSIDADE DO PORTO \\ Porto, Portugal \\ Tel.: $+351 /(22)-5081808$. \\ Fax: $+351 /(22)-5081443$. \\ ** INSTITUTO POLITÉCNICO DE BRAGANÇA, \\ Bragança, Portugal \\ Tel.: $+351 /(273)-303158$. \\ Fax: $+351 /(273)-313051$ \\ E-Mail: raraujo@fe.up.pt,hteixeira@ipb.pt,jbarbosa@ipb.pt and avtl@ipb.pt \\ URL: http://www.fe.up.pt, www.ipb.pt
}

\section{Keywords}

«Education tool», «Teaching», «Induction motor», «Adjustable speed drive»

\begin{abstract}
This paper presents a digital controller suitable for an instructional laboratory in electric drives. A prototype has been designed specifically to meet the requirements of low cost and motivating education tool, and it contains all of the active functions required to implement the open loop control of induction motors. This approach develops the concept of "grey box" module where the details are built for convenience, but not hidden inside as a "black box". Introductory laboratory experiments are presented to demonstrate how this hardware can be integrated in laboratory classes to present the most important concepts as regards adjustable speed drives.
\end{abstract}

\section{Introduction}

The need for power converters is to increase dramatically in the coming years. The increase of the energy consumption and environmental legislation are forcing the development of an ever increasing number of electric cars and the utilization of clean renewable energy resources, such as wind and solar generators, requiring power conditioning on their outputs. In this scenario, the technology of power electronics and electric drives is the core of clean technologies. Consequently, it should have a core place in the formation of electrical engineers. However, the experience has shown that power electronics and drives is a hard task for under graduated students, mostly because of the maturity that the subject demands. Students are not often accustomed to assimilate material from different subjects at one time thereby making it difficult for them to simultaneously bring together the electrical circuit analysis, analog and digital electronics, electric and magnetic analysis and control considerations which are required to fully describe an electric drive. As a solution to this challenge, this paper discusses the development of a hardware board based on the MC3PHAC motor controller and a power inverter that allows students to easily visualize the motor during motion, vary parameters of the controller to observe their effects on the performance, and gain experience with induction motor control.

Education methodologies and educational tools in the field of power electronics and electric drives have been a topic of considerable debate over the last decade and still remain a topic of current discussion [1-3]. How to teach students with different sensitivities and motivations is an often-asked question. Based on the 
author's teaching experience a different teaching methodology has been developed in order to enable students to learn quicker and easier by increasing their motivation. The traditional feeding teaching technique was changed and students are invited to build and use "toys" with which they can practice and see how "things" are made and understand how they work [4]. This approach uses the concept of "grey box" which allows students to start immediately on experimental work and observe the internal hardware of an electric drive while it is operating. The hardware setup is simple to build and inexpensive when compared with other conventional options. This approach permits that students are motivated to understand the inner workings - the core of any real power converter.

Web based laboratory tools have become the centre of attention recently [5]. The virtual power laboratory can be a valuable supplement to formal lecture allowing students to experiment with several scenarios. The disadvantage of a virtual laboratory is the obvious lack of student physical contact with the devices under study. A significant drawback of hardware laboratory works is the considerable effort required to build a complete electric drive system. Power inverter, gate drivers, galvanic isolation, control functions, layout, among other issues must be addressed before the experimental work could be done. This work is intended to be a contribution to stimulate the student's interest in power electronics and electric drives as well as to provide them with skills in measurement and instrumentation. The ability to correctly interpret and verify results is important not only for electronic design, but also in other work areas where engineers are increasingly relying on industrial systems developed by others. The main goal of the laboratory is to give the possibility to the students to put in practice the theory without spending too much time with details concerning to sophisticated hardware implementations without need of high level and expensive development systems like in $[6,7]$.

\section{Motivation}

In this work a low cost solution is presented to develop an induction motor control prototype to be used in the laboratory, which is easy and safe to handle, manipulate and test. The purpose of this prototype is not centered on achieving a great dynamic performance of the induction motor control, but on highlighting the main concepts of this issue and leading the students to play with the system and practice in laboratory measurements. In addition, this prototype has the advantage that the explanations are made with a real and concrete system and it is a resource for students to improve the skills that are required to analyze and design electric drive systems. The novel hardware includes a power inverter, gate-drive boards, voltage and current sensors, and interface and controller boards.

The design process of this hardware had different objectives when compared with a typical induction motor drive design. Rather than a design to meet specific performance requirements, a design to meet general educational objectives was developed. The design and details are freely available to educators who wish to apply this approach.

The purpose of the laboratory is to familiarize students with power electronics and their functions as used in motion control. A laboratory for electric drives consists of some experiments carried out in a 2-3 hour session. The students work in groups of two or three students. They have to prepare the laboratory using its handouts. Typically, the handouts for the experiments provide an equipment list and a list of topics which can be explored with the prototype hardware. The students can use a converter that can implement nearly any dc-dc or dc-ac converter, providing students with a means to quickly begin to explore various experiments [8]. The key improvement is a new "grey box" induction motor control using the MC3PHAC motor controller from Motorola. The "box" contains an inexpensive microcontroller from Atmel that controls the MC3PHAC.

The motivation to have developed this hardware is manifold. It provides motivation to the students since it is a piece of hardware which they can easily understand. It improves their design skills regarding open 
loop voltage/frequency control by putting in practice the theoretical concepts from the lectures with regard to induction motor control. They gain experience with the design of a prototype in the same way as it would be done on the job in an industrial environment. They have a real feeling of satisfaction at the end of the laboratory work because they have opportunity to do something that "move". Finally, a digital electronic hardware realization uses the know-how of digital systems and electronics already acquired by the students in previous subjects.

\section{Description of the prototype hardware}

It is essential to the "grey-box" concept that there are not any hidden functions or control actions. As stated above it has been conceived with the main purpose of full integration in laboratory experiments for the teaching of electric drives concepts and methods. The control of an induction motor requires pulsewidth-modulated control of the six switches of a three-phase power voltage inverter. The control law can be implemented in open loop with no speed or position feedback. The $V / f$ ratio is maintained constant to provide a flux constant over the operating range into nominal frequency. The hardware prototype has been developed to allow the contact of the student with a real solution of three-phase induction motor control. We note that open loop voltage/frequency control is used in numerous industrial applications where the requirements related to the dynamic performance of drive control are of secondary importance.

The complete diagram of the integrated prototype is shown in fig. 1. The main modules of the setup are: the controller board based on the MC3PHAC motor controller, the master board, a set of gate driver boards, the power converter and the induction motor loaded by a powder break. The power converter used in this work was built using some modules of the generic converter described with detail in [8] which was also designed as a "plug and play" set of pieces.

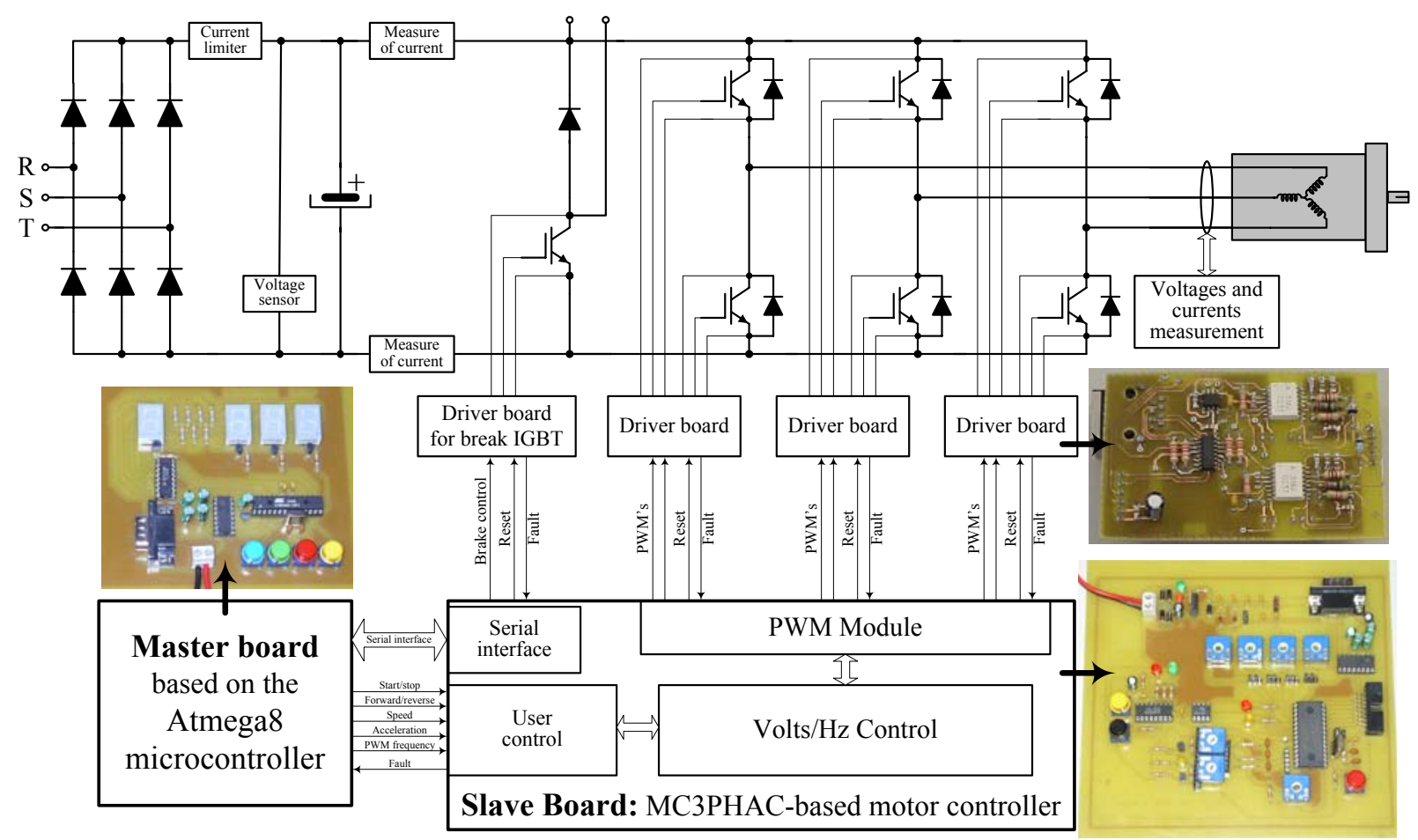

Fig. 1: Diagram of the global hardware integrated setup developed as education tools for electric drive systems. 


\section{Motor controller board}

The controller board is based on the MC3PHAC chip from Motorola, illustrated in fig. 2. The MC3PHAC is a motor controller designed to meet the requirement of low cost solution for induction motor control applications [9]. The device contains all of the functions required to implement the open loop voltage/frequency control of a voltage inverter fed induction motor. The main device features are summarized as follows:

1. A three-phase pulse-width modulator to generate six center-aligned PWM's in three groups of complimentary PWM's (with dead-time);

2. An open loop voltage/frequency speed control with possibility to adjust of the low-speed voltage boost;

3. The forward/reverse rotation and start/stop commands;

4. The system fault input;

5. A serial communications interface.

The MC3PHAC motor controller operates in either of two modes: Stand-alone or external master mode. Mode selection occurs at power-up as specified by state of pin 20. In stand-alone mode the operating parameters are configured during power-up via passive components connected to the device. In the second mode of operation the MC3PHAC is configured by software via an external master.

\section{Master board}

A key improvement in this work is the implementation of an inexpensive 8-bit microcontroller that uses the serial interface in master mode to control the MC3PHAC. The master software commands were implemented in the Atmega8 microcontroller from Atmel Corporation. A view of the master board is shown in fig. 3 . The Atmega8 has $8 \mathrm{~K}$ bytes of In-system programmable Flash, 512 bytes of EEPROM, $1 \mathrm{~K}$ byte of internal random access memory, a six-channel multiplexed 10b A/D converter, 23 digital I/O lines and a bidirectional serial interface.

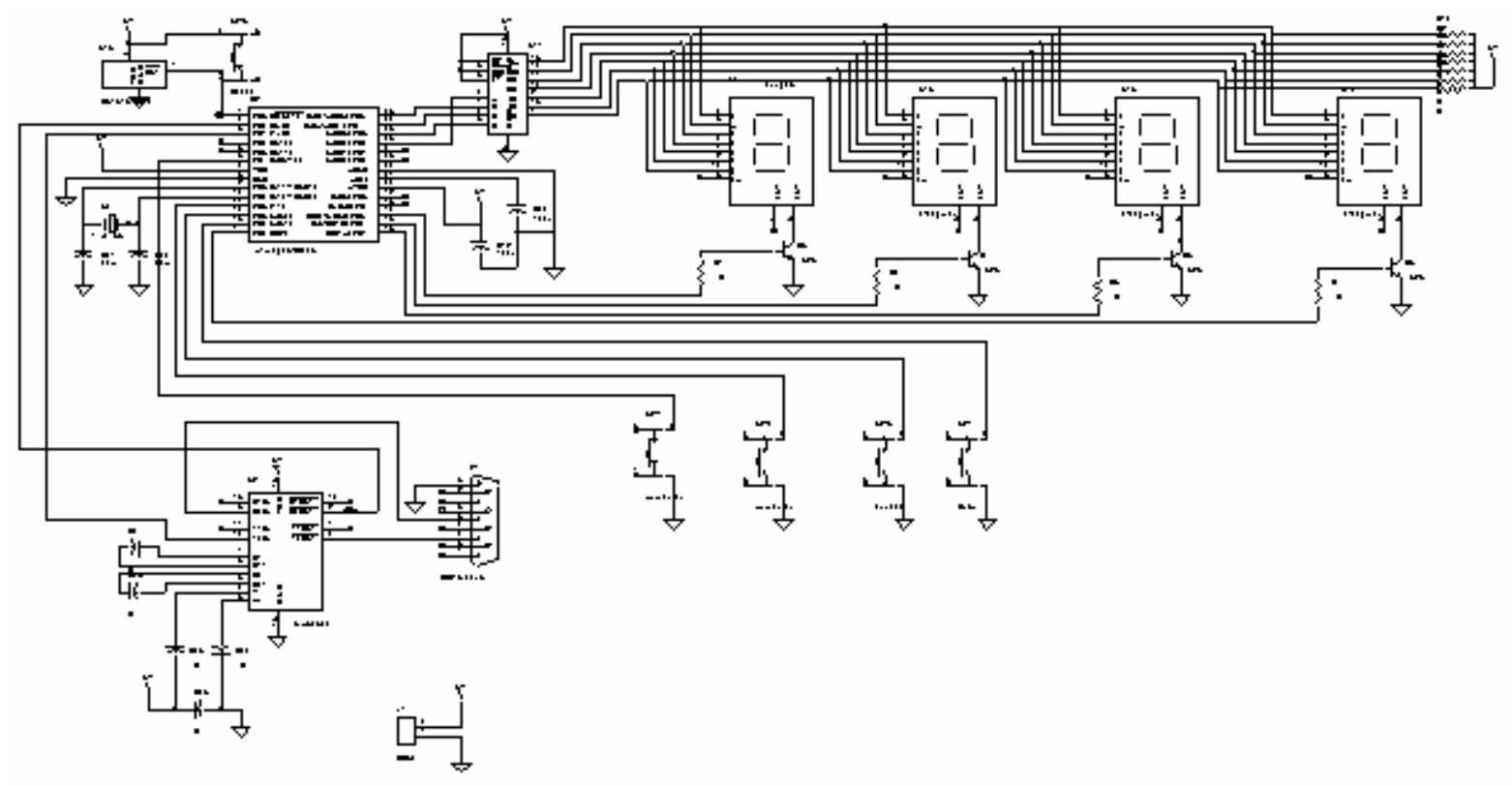

Fig. 2: Circuit diagram for the master board based on the Atmega8 microcontroller. 
Understanding how the devices are connected is straightforward. A serial communication cable connects the devices. The requirements of the serial port are as follows: communication at 9600 baud rate, rows of 8 bits, and no parity. The serial communication between the Atmega8 and MC3PHAC is based on a master-slave protocol, where the master sends a message with a command and its arguments, and the MC3PHAC returns the data and the operation status code. In the protocol, two data packets are defined: the command packet and the response packet. In other words, the basic communication mechanism for the devices is that the master sends out a command packet and waits for a response packet from the MC3PHAC. The protocol also defines valid commands between master and slave devices. The MC3PHAC gives the possibility of access to a set of twenty-five variables that can be used to control or monitor the state of the motor. A complete description of the protocol can be found in [9]. All programming is done in $\mathrm{C}$ language. The schematic diagram showing the Atmega8 microcontroller is shown in fig. 2.

The main advantages of this prototype hardware are summarized as follows:

1. The laboratory setup is assembled from inexpensive modules and it is not necessary a personal computer to realize the control functions;

2. The parameters of the MC3PHAC motor controller can be easily changed;

3. The students can make changes and observe the results in real-time using an oscilloscope;

4. The setup is part of a set of integrated education tools enabling the students to manage hardware, learn and validate concepts based on experimentation;

5. The hardware is suitable to analyze the basic control of induction motors, such as starting, braking, and speed inversion.

\section{Examples of experiments}

The purpose of this hardware setup is to familiarize the students with frequency converters and their functions as used in variable speed induction motor control applications. The experiments below support the following goals: familiarize students with induction motor drives, learn about $V / f$ control and visualize the typical experimental waveforms in power stage. The experiments are being designed to implement the voltage/frequency control of induction motor. To demonstrate the usefulness and benefits of the prototype hardware some typical signals are shown.

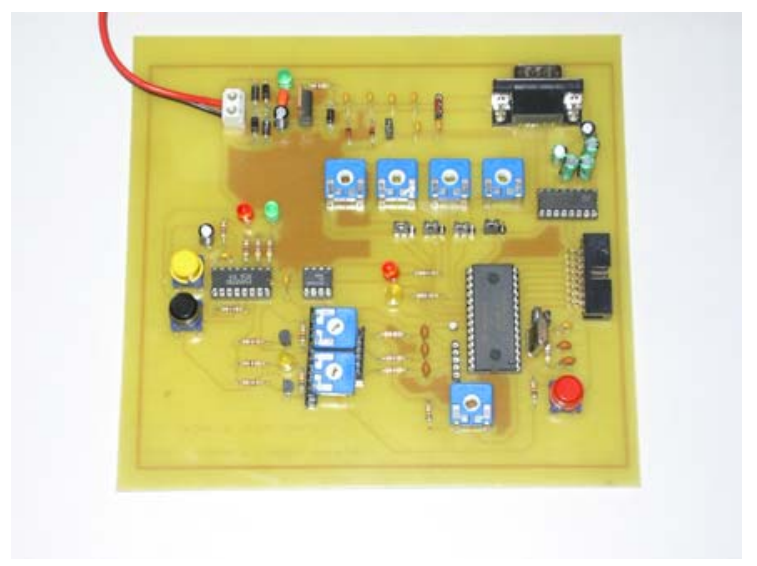

Figure 3: The controller board based on MC3PHAC.

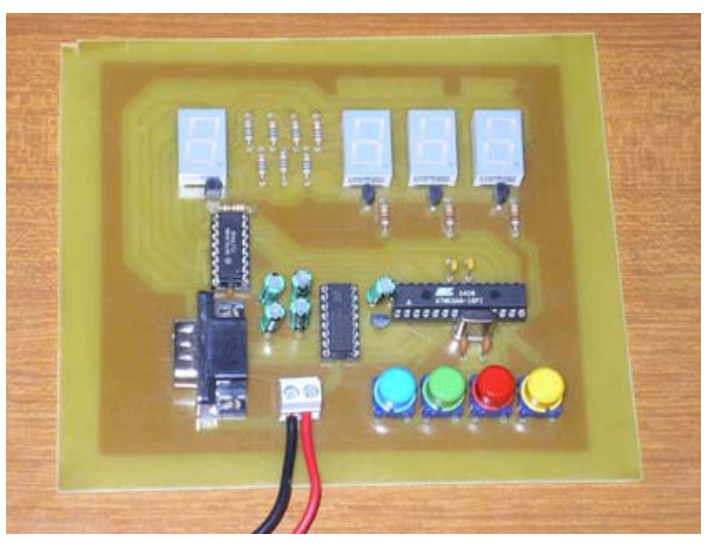

Figure 4: The master board using the Atmega8. 


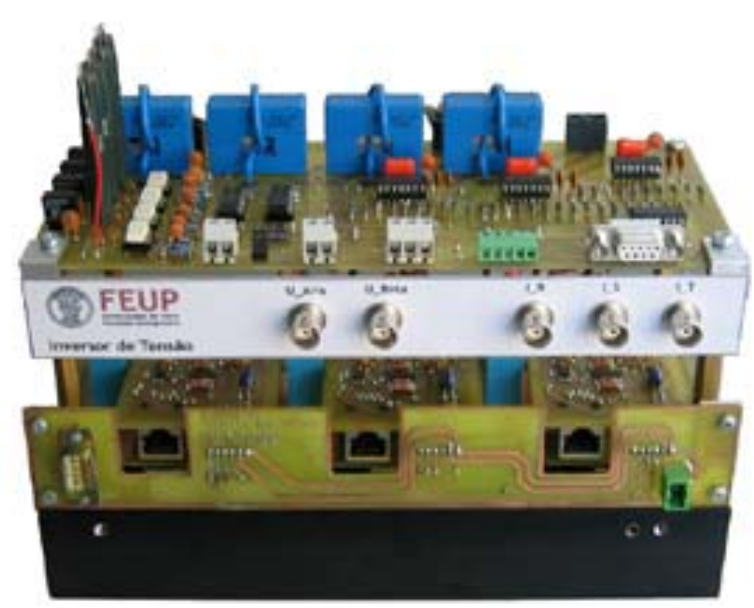

Figure 5: Voltage source inverter and measurement board implemented as described in [8].

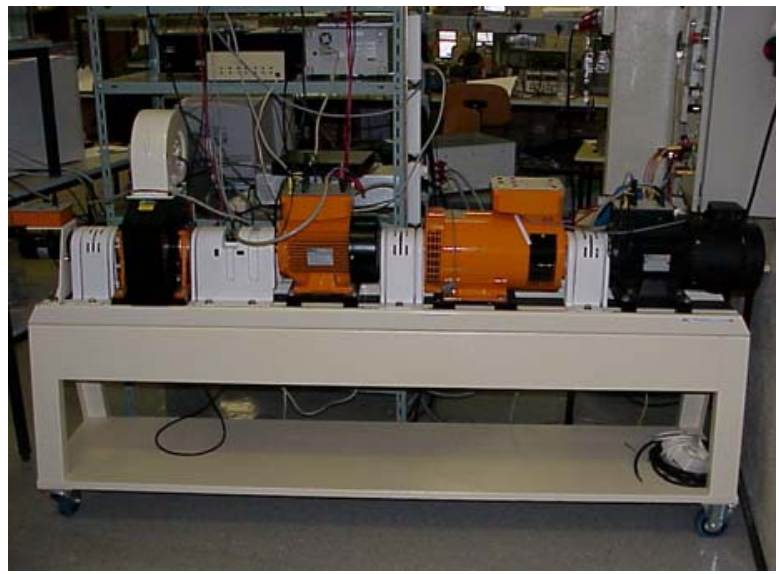

Figure 6: The experimental test bench.

Generically speaking, the control of a three-phase induction motor requires pulse-with modulated control of the six switches of a three-phase power inverter. The six switches form a three-leg inverter, which can be used to connect the leg of a winding to the positive or the negative DC bus. The two switches on the same leg must never be ON simultaneously, otherwise the positive and negative buses would be shortcircuited. If one switch is ON, then the other must be OFF, thus they are driven as complementary pairs. It should also be noted that the typical switch devices (in this case IGBTs) used in the inverter often require more time to turn off than to turn on. For this reason, a minimum dead-time must be inserted between the off and on time of complimentary channels.

The MC3PHAC generates six center-aligned PWMs in three groups of complimentary PWMs (see figure 7). This allows the MC3PHAC to be connected directly to the interface board. Note that the MC3PHAC will not allow the PWM signals to be activated until certain critical parameters have been communicated, such as PWM polarity and dead-time. Fundamental discussion in practical inverters is the issue of deadtime, which is inserted between the off and on-times of each complementary signal pair and may be adjusted to any value between 0 and $32 \mathrm{~ms}$ (see figure 8). Another important aspect is the possibility of changing the gate resistance so that students can study the device rise times. The gate-driver boards are connected to the semiconductor devices using small circuits boards with terminal blocks and connectors. These boards are placed atop the devices, between the snubber circuits, simplifying dramatically the wiring.

Figure 9 shows the line-to-line voltage and current waveforms taken from a $3 \mathrm{~kW}$ induction motor driven by the voltage sorce inverter connected to a three-phase 400VAC, $50 \mathrm{~Hz}$ net. Figure 10 shows the stator current and shaft speed during a slow reversing test.

\section{Conclusion}

This paper has introduced the concept of "grey-box" hardware modules as education tools and described a new controller board for induction motors in this context. A first link between an educational system and a real technical or industrial system is established, which enhances the student's skills to measure and interpret measurements properly and compare them to theoretical predictions made by simulations tools. 


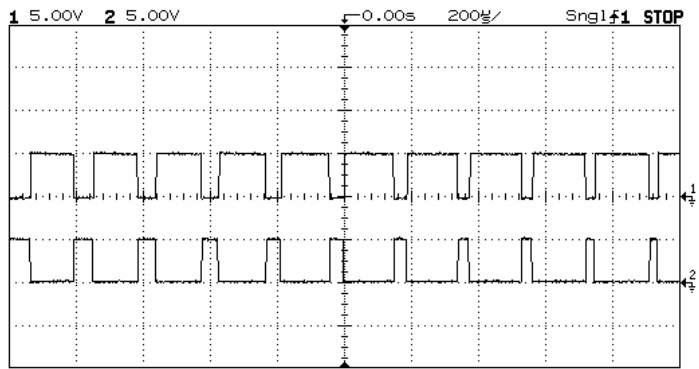

Figure 7: Typical drive signals for switches of the one leg inverter.

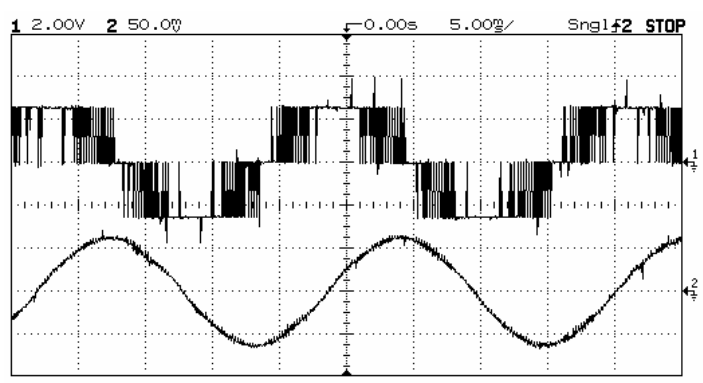

Figure 9: The inverter output line-to-line voltage and stator current of the induction motor at 1360 rpm.

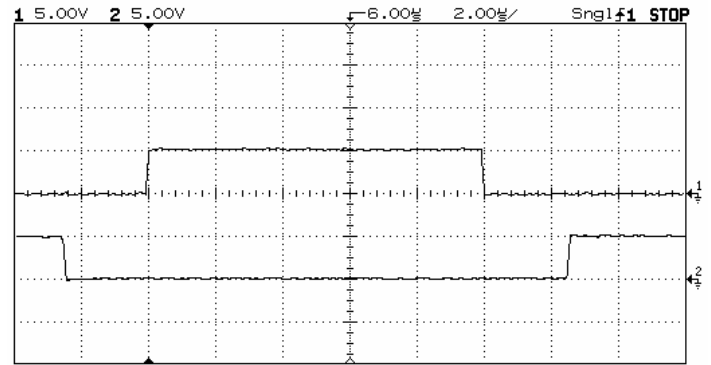

Figure 8: Visualization of the dead-time in one leg of the inverter.

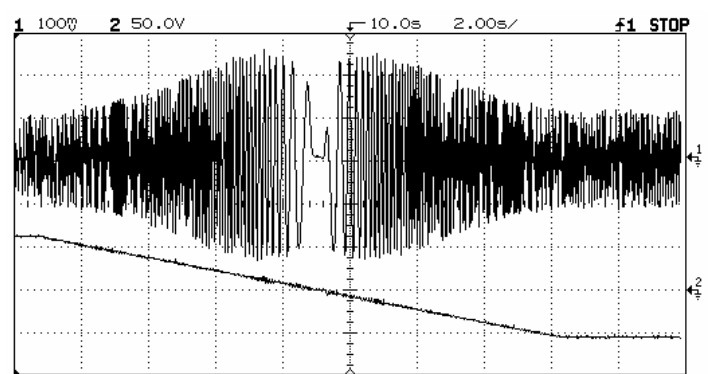

Figure 10: Experimental oscillogram illustrating slow speed reversal. Phase current in channel 1 and speed in channel 2

In the instructional environment we have found that the controller board helps to solidify fundamental concepts such as three-phase PWM generation, dead-time, isolated gate drivers and basic control. Another additional advantage of the realized hardware is its low price when compared to commercial "black-box" module of power laboratory equipment. Regarding the disinterest of students by Power Electronics and Electric Drives, the authors have described their current methodology regarding laboratory classes to motivate students and captivate new ones into this domain by using the proposed controller system to play with work aiming a practical understanding and exercising of the fundamental concepts.

\section{References}

[1]. C. Fernández, O. García, J. A. Cobos,J. Uceda, Hardware and Software Environment for Self-learning in Power Electronics, $10^{\text {th }}$ International Power Electronics and Motion Control Conference, EPE-PEMC 2002, Croatia, September, 2002.

[2]. T. Undeland, N. Mohan, Meeting the Challenge: Revitalizing courses in Power Electronics and Electric Drives, Nordic Workshop on Power and Industrial Electronics, NORPIE 2002, Sweden, August, 2002.

[3]. P. Barrade, A. Rufer, Teaching Power Electronics: stiring up the interest and methodology of teaching with contribution of new technologies, EPE-E $=\mathrm{TeM}^{2}$, Tomorrow's Education in Electrical Technologies revisited Methods \& Tools for renewed Motivation, Belgium, March, 2001.

[4]. R. E. Araújo, V. Leite, D. Freitas, A Low Cost Solution for Laboratory Experiments in Induction Motor Control, in Proceedings of 10th European Conference on Power Electronics and Applications - EPE 2003, September 24, Toulouse, France, 2003.

[5]. U. Drofenik, J. W. Kolar, Survey of Modern Approaches of Education in Power Electronics, in Proceedings of Applied Power Electronics Conference and Exposition, 2002. APEC 2002. Seventeenth Annual IEEE, Volume: 2, 10-14 March, 2002. 
[6]. R. Teodorescu, M. Bech, A. Jorgensen, K. Larsen, F. Blaabjerk, J. Pedersen, Advanced Prototyping Tools for Project- and Problem-Based Learning, in Proc. EPE-PEMC02, Dubrovnic \& Cavtat, Croatia, July, 2002.

[7]. R. Teodorescu, M. Bech, A. Jorgensen, K. Larsen, F. Blaabjerk, J. Pedersen, A New Approach in Teaching Power Electronics Control of Electrical Drives using Real-time Systems, in Proc. The 7th Workshop on Computers in Power Electronics, 2000. COMPEL 2000, July, 2000.

[8]. Vicente Leite, José Barbosa, Henrique Teixeira, Rui E. Araújo, A Generic Converter for Experimentation Based Power Electronics Learning, in Proceedings of 11th European Conference on Power Electronics and Applications - EPE 2005, September 11-14, Dresdem, Germany, 2005

[9]. Freescale Semiconductor, Inc., MC3PHAC Motor Controller -General-purpose 3-phase AC industrial motor controller: Designer Reference Manual, April, 2002. 